\title{
Peptidylglycine a-Amidating Monooxygenase And Adrenomedullin Measurement In Cirrhosis Suggesting Cardiomyopathy
}

Jens P. Goetze ( $\sim$ JPG@dadlnet.dk )

University of Copenhagen

\section{Andrei Voiosu}

Department of Clinical Physiology and Nuclear Medicine

\section{Signe Wiese}

Gastro Unit, Medical Division, Hvidovre Hospital, Hvidovre, Denmark

Janin Schulte

SphingoTec GmbH

\section{Paul Kaufmann}

PAM Theragnostics $\mathrm{GmbH}$

\section{Andreas Bergmann}

SphingoTec $\mathrm{GmbH}$

\section{Emil D. Bartels}

University of Copenhagen

\section{Søren Møller}

Department of Clinical Physiology and Nuclear Medicine

\section{Research Article}

Keywords: Adrenomedullin, cirrhosis, heart failure, proadrenomedullin, peptidylglycine a-amidating monooxygenase.

Posted Date: November 30th, 2021

DOI: https://doi.org/10.21203/rs.3.rs-1006070/v1

License: (c) (i) This work is licensed under a Creative Commons Attribution 4.0 International License. Read Full License 


\section{Abstract}

Peptidylglycine a-amidating monooxygenase (PAM) is a processing enzyme involved in maturation of regulatory peptides. One product of PAM activity is adrenomedullin (bio-ADM), which regulates vascular tone and endothelial integrity. In this study, we examined PAM activity and bio-ADM concentrations in patients with various degrees of hepatic cirrhosis including the role of the liver in net release of the two markers. We enrolled 48 patients with cirrhosis and 16 control subjects: The patients were evenly distributed according to the Child-Turcotte classification. PAM activity was progressively increased in cirrhotic patients but without a net release across the liver, leg, or kidney. In contrast, bio-ADM concentrations were not only associated to severity of disease but also found to be directly released by the liver. Given the major expression of PAM in the heart, we propose that increased PAM activity in plasma from patients with cirrhosis may reflect cardiac involvement, e.g. cirrhotic cardiomyopathy.

\section{Introduction}

Peptidylglycine a-amidating monooxygenase (PAM) is a processing enzyme involved in maturation, e.g. C-terminal amidation, of a plethora of regulatory peptides. Besides its systemic expression in neuroendocrine tissue, PAM is dominantly expressed in the heart ${ }^{1}$. One product of PAM activity is the amidated hormone adrenomedullin (bio-ADM), which regulates vascular tone and endothelial integrity ${ }^{2}$. Albeit adrenomedullin was named after the adrenals, the tissue profile of expression involves the endothelium in most organs, including the liver.

Now, PAM activity can be quantitated in circulation, e.g. in patient plasma, where PAM activity may represent a new marker of disease. So far, no condition has been examined specifically, including cirrhosis or cardiac dysfunction. For bio-ADM, scarce data are available from patients with septic shock or heart failure ${ }^{3,4}$, and a singular report has associated bio-ADM concentrations with cirrhosis ${ }^{5}$. Given the fact that cirrhosis can cause cardiomyopathy ${ }^{6}$, we examined both PAM activity and bio-ADM concentrations in plasma from patients with various degrees of cirrhosis. The specific aim was to test whether the markers in plasma are related to severity of disease and hepatic release/clearance. Notably, these studies have only recently become possible due to development of new methodology for quantitating PAM activity and bio-ADM concentrations in plasma.

\section{Methods}

We enrolled 48 patients with cirrhosis and 16 control subjects. The patients were evenly distributed according to the Child-Turcotte classification (16 patients in class A, 16 patients in class B, and 16 patients in class $\mathrm{C}$ ). After an overnight fast, the patients underwent a complete hemodynamic examination with catheterization of the liver, renal, and femoral veins and corresponding arteries, from where blood was simultaneously collected. The plasma was stored at $-80^{\circ} \mathrm{C}$ until biochemical analyses. PAM activity was determined in heparin plasma with a new assay from PAM Theragnostics $\mathrm{GmbH}$ (Hennigsdorf, Germany). PAM activities are reported as U/L by measuring the amount of bio-ADM 
generated from glycine-extended ADM by plasmatic PAM. One Unit $(U)$ is defined as $1 \mu \mathrm{g}$ bio-ADM produced within 1 hour (Kaufmann et al., submitted). Bio-ADM was measured at a research laboratory at SphingoTec $\mathrm{GmbH}$ (Hennigsdorf, Germany) using a method previously described ${ }^{7}$. Intra- and interassay variation was $<10 \%$ and $<8 \%$, respectively. In 200 healthy adults, the median concentration has earlier been reported to be $20.7 \mathrm{pg} / \mathrm{mL}^{8}$. All data (without personal data that can be referred to the individual patients) and results can be requested from the corresponding author. All patients gave informed (oral and written) consent to participate in the study, which was approved by the regional ethics committee in Copenhagen, Denmark (de videnskabsetiske komitéer i Region Hovedstaden). The study was performed in accordance with all relevant guidelines and regulations.

\section{Results}

First, we assessed whether PAM activity was related to severity of cirrhosis (Figure 1A). Patients with Child $\mathrm{C}$ cirrhosis displayed increased PAM activity in circulation. PAM activity across the liver, however, did not reveal any release or extraction of PAM activity $(p=0.93$, Figure $1 B)$, nor from the kidney $(p=$ $0.57)$ or leg $(p=0.09)$ (data not shown). In addition, there was no association of known clinical manifestations of cirrhosis, e.g. ascites $(p=0.58)$, esophageal varices $(p=0.55)$ or portal hypertension ( $p$ $=0.14$ ). For established biochemical markers in cirrhosis, we did find associations of PAM activity to alanine aminotransferase $(r=0.30, p=0.04)$, bilirubin $(r=0.47, p=0.001)$, INR $(r=0.42, p=0.004)$, but not to albumin $(p=0.07)$ or alkaline phosphatase $(p=0.4)$.

We assessed the same associations for bio-ADM concentrations, where a marked difference between the different stages of cirrhosis was noted (Figure 1C). We also checked for associations between the bioADM concentrations in femoral vein samples and standard parameters of hepatic function. For the prothrombin time and albumin concentrations, negative correlations were found $(r=-0.35, p=0.02, r=$ $-0.54, p=0.0001$, Figure 1D). We then examined whether the cirrhotic liver release bio-ADM to circulation. A net release was identified across the liver (hepatic artery: 13.0 (8.7-21.0) vs. hepatic vein: 19.7 (12.729.6) $\mathrm{pg} / \mathrm{mL}, \mathrm{n}=48, \mathrm{p}<0.001$ ) (Figure $1 \mathrm{E}+\mathrm{F}$ ). There was no release or extraction across the kidney, while a release across the lower limp was registered (femoral artery: 12.6 (7.6-22.0) vs. femoral vein: 21.8 (13.529.2) $\mathrm{pg} / \mathrm{mL}, \mathrm{n}=48, \mathrm{p}<0.001)$. We further tested whether bio-ADM concentrations in plasma reflect severity of cirrhosis as defined by the Child-Turcotte score. A marked increase in bio-ADM concentrations in all vascular beds was found when comparing patients in class A to those in classes B or C. Finally, we evaluated whether bio-ADM concentrations reflect complications of cirrhosis as defined by the presence of portal hypertension and/or ascites. For all vessels, bio-ADM concentrations were increased in patients with ascites $(n=26)$ compared to patients without $(n=22)$; in the femoral vein, the difference was 1.5fold $(p=0.05)$. In contrast, there was no difference between patients with esophageal varices $(n=32)$ compared to patients without $(n=16)$.

\section{Discussion}


Here, we are able to report on peptidylglycine a-amidating monooxygenase (PAM) activity combined with adrenomedullin (bio-ADM) concentrations in plasma from patients with cirrhosis. PAM activity was progressively increased in cirrhotic patients but without a net release across the liver, leg, or kidney. In contrast, bio-ADM concentrations were not only associated to severity of disease but also found to be released by the liver. Taken together, our data suggest that PAM activity does not directly reflect the cirrhotic liver but rather another organ or a systematically upregulation of PAM release into plasma. In this context, it is worth noting that PAM is predominantly expressed in the heart ${ }^{1}$, and we speculate that the increased PAM activity in cirrhosis reports on cardiac involvement, e.g. cirrhotic cardiomyopathy. While the relation between increased PAM activity and cardiomyopathy must be confirmed in patients with primary heart disease, it opens up for a new marker of cirrhotic cardiomyopathy. Moreover, increased PAM activity in circulation may be more than a marker of disease, as PAM amidates, i.e. activates, a plethora of regulatory peptides.

The data on bio-ADM in circulation extends earlier data from cirrhotic patients by showing that the liver is a true site of bio-ADM release. Importantly, the release of bio-ADM from the liver not only affects local vasculature but also the systemic circulation. Generally, bio-ADM relaxes the pulmonary vasculature and induces systemic hypotension; a common feature seen in patients with advanced cirrhosis. Whether the hepatic gradient of bio-ADM correlates with pulmonary artery pressures remains to be established. It is noteworthy, however, that we earlier reported that bio-ADM concentrations in patients with advanced heart failure are associated with pulmonary pressures, and that the concentrations in plasma are related to the degree of hepatomegaly and the presence of ascites/edema ${ }^{3}$. This suggests a common pathogenic link between heart and liver failure ${ }^{6}$. We conclude that PAM activity in plasma is increased in cirrhosis and may reflect cirrhotic cardiomyopathy. Bio-ADM is also increased in cirrhosis and seems mainly to reflect severity of hepatic disease. Using these two measures in combination might provide a new tool identifying patients at risk of developing cirrhotic cardiomyopathy and may help to better understand the mechanisms involved in the disease.

\section{Declarations}

Author Contributions: SM: Study concept and design, acquisition of data, analysis and interpretation of data, and revision of manuscript. AV: analysis and interpretation of data, and revision of manuscript. SW: Acquisition of data, analysis and interpretation of data, and revision of manuscript. EDB: Interpretation of data and revision of manuscript. JPG: Analysis and interpretation of data, writing of manuscript. JS, PK, and AB: Analysis of blood and revision of manuscript.

Competing Interest Statement: Drs. Schulte and Bergmann are affiliated to SphingoTec GmbH, the commercial manufacturer of the bio-ADM methodology. Paul Kaufmann and Dr. Bergmann are affiliated to PAM Theragnostics $\mathrm{GmbH}$, the commercial manufacturer of the PAM methodology. All other authors have no conflicts of interest to declare. 


\section{References}

1. Bäck, N., Luxmi, R., Powers, K. G., Mains, R. E. \& Eipper, B. A. Peptidylglycine a-amidating monooxygenase is required for atrial secretory granule formation. Proc. Natl. Acad. Sci. USA. 117, 17820-17831 (2020)

2. Voors, A. A. et al. Adrenomedullin in heart failure: pathophysiology and therapeutic application. Eur. J. Heart Fail.21,163-171(2019)

3. Goetze, J. P. et al. Bioactive adrenomedullin in plasma is associated with biventricular filling pressures in patients with advanced heart failure.Eur. J. Heart Fail.23,489-491(2021)

4. Mebazaa, A. et al. Circulating adrenomedullin estimates survival and reversibility of organ failure in sepsis: the prospective observational multinational Adrenomedullin and Outcome in Sepsis and Septic Shock-1 (AdrenOSS-1) study. Crit. Care22,354(2018)

5. Kimer, N., Goetze, J. P., Bendtsen, F. \& Møller, S. New vasoactive peptides in cirrhosis: Organ extraction and relation to the vasodilatory state.Eur. J. Clin. Invest.44,441-452(2014)

6. Møller, S., Danielsen, K. V., Wiese, S., Hove, S. \& Bendtsen, F. J. D. \& An update on cirrhotic cardiomyopathy.Expert Rev. Gastroenterol. Hepatol.13,497-505(2019)

7. Weber, J. et al. Sandwich immunoassay for bioactive plasma adrenomedullin.J. Appl. Lab. Med.02,222-233(2017)

8. Marino, R. et al. Plasma adrenomedullin is associated with short-term mortality and vasopressor requirement in patients admitted with sepsis.Crit. Care18,R34(2014)

\section{Figures}

\section{Figure 1}

Panel A shows the PAM activity in the femoral vein accordingly to severity of cirrhosis. Norm represents healthy individuals. Groups were compared with Kruskal-Wallis test using Dunn's multiple comparison. Panel $B$ shows the PAM activity across the liver. Data were analyzed using Wilcoxon testing. Panel C shows the bio-ADM concentrations according to disease severity: Norm represents healthy individuals. Groups were compared with Kruskal-Wallis test using Dunn's multiple comparison. Panel D shows linear regression analyses comparing bio-ADM (from the femoral vein) concentrations with measurements of albumin and prothrombin time. One patient was excluded (extreme outlier). Panel $\mathrm{E}$ and $\mathrm{F}$ show the bioADM gradients across the liver in 48 patients (cirrhosis) and 16 control subjects (normal). Data were analyzed using Wilcoxon testing. One patient was excluded (extreme outlier). 